\title{
Papulonecrotic Tuberculid of the Penis
}

\author{
Sheetanshu Kumar ${ }^{1} \quad$ Ankur Guliani ${ }^{1} \quad$ Divya Aggarwal ${ }^{2} \quad$ Dipankar De $^{1}$
}

${ }^{1}$ Department of Dermatology, Venereology and Leprology, Postgraduate Institute of Medical Education and Research, Chandigarh, India

${ }^{2}$ Department of Histopathology, Postgraduate Institute of Medical Education and Research, Chandigarh, India

Ann Natl Acad Med Sci (India) 2020;56:46-47

A 51-year-old male presented with multiple asymptomatic nonhealing ulcers over the glans penis for last 3 months. He was a heterosexual individual with no history of genital ulcers in the life partner. There was no history of extramarital sexual contact. Patient denied any history of trauma, fever, cough, constitutional features, and drug intake. On examination, there were multiple tender, mildly indurated, punched out ulcers with irregular margins, and yellowish white necrotic slough on the floor, varying in size from $1 \mathrm{~cm} \times 1 \mathrm{~cm}$ to $0.5 \mathrm{~cm} \times 0.5 \mathrm{~cm}$ with areas of depressed varioliform and irregular pitted scarring ( - Fig. 1). There was no inguinal lymphadenopathy.

The Mantoux test was strongly positive $(26 \mathrm{~mm} \times 21 \mathrm{~mm}$; -Fig. 2). Urine examination for acid fast bacilli was negative. Ultrasound examination of genitourinary system, chest X-ray, contrast-enhanced computed tomography chest was not contributory. Histopathological examination revealed epidermal thinning and necrosis with granulomatous infiltrate composed of lymphocytes and macrophages. Ziehl Neelsen stain for acid fast bacilli was negative. Diagnosis of
Address for correspondence Dipankar De, MD, Department of Dermatology, Venereology and Leprology, Postgraduate Institute of Medical Education and Research, Sector 12, Chandigarh 160012 , India (e-mail: dr_dipankar_de@yahoo.in).

papulonecrotic tuberculid of penis was made on the basis of history, clinical examination, histopathological examination, and laboratory investigations. Antitubercular therapy was started for 6 months (isoniazid, rifampicin, pyrazinamide,

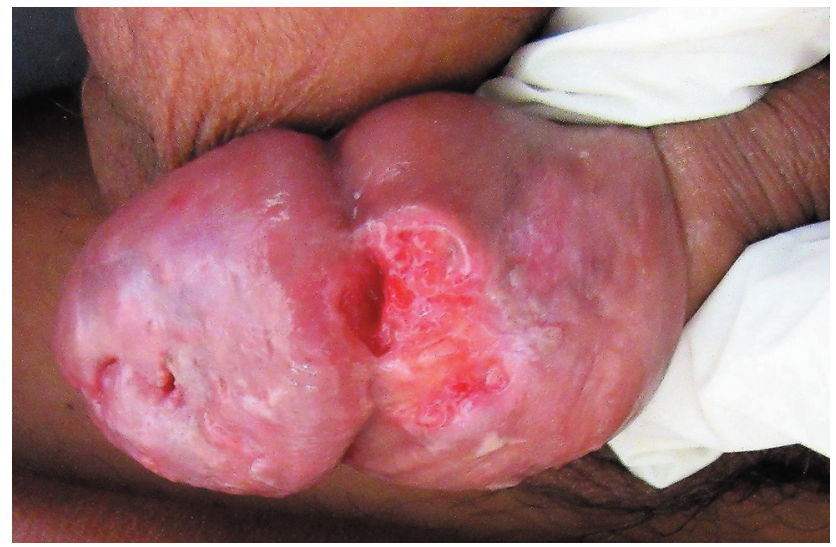

Fig. 1 Multiple ulcers on the coronal sulcus and neck of glans with irregular margins and granulation tissue at the floor.

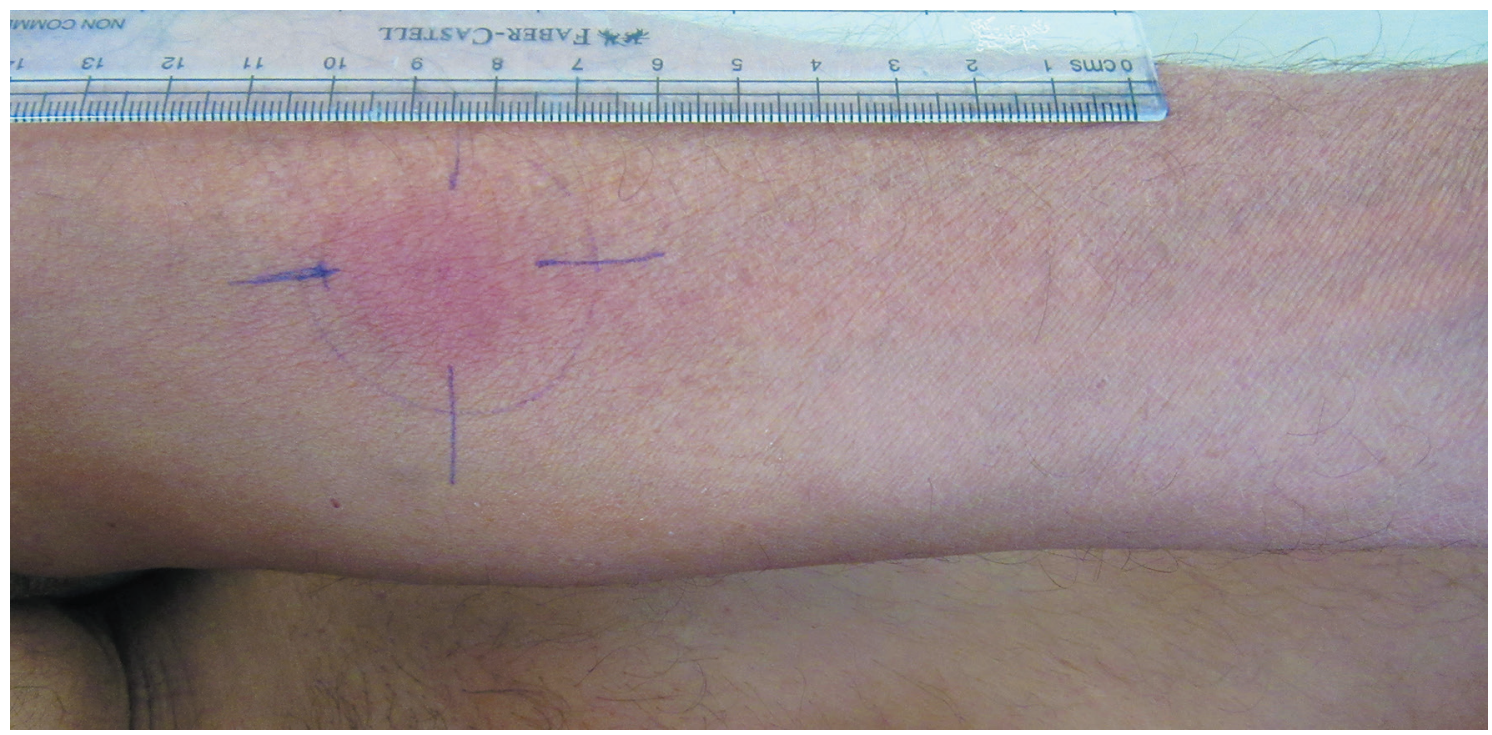

Fig. 2 Mantoux's test performed on volar aspect of forearm was strongly positive $(26 \mathrm{~mm} \times 21 \mathrm{~mm})$.

DOI https://doi.org/ 10.1055/s-0040-1710196 ISSN 0379-038X.
License terms

(ㅇ) (1) $\ominus \circledast$ 


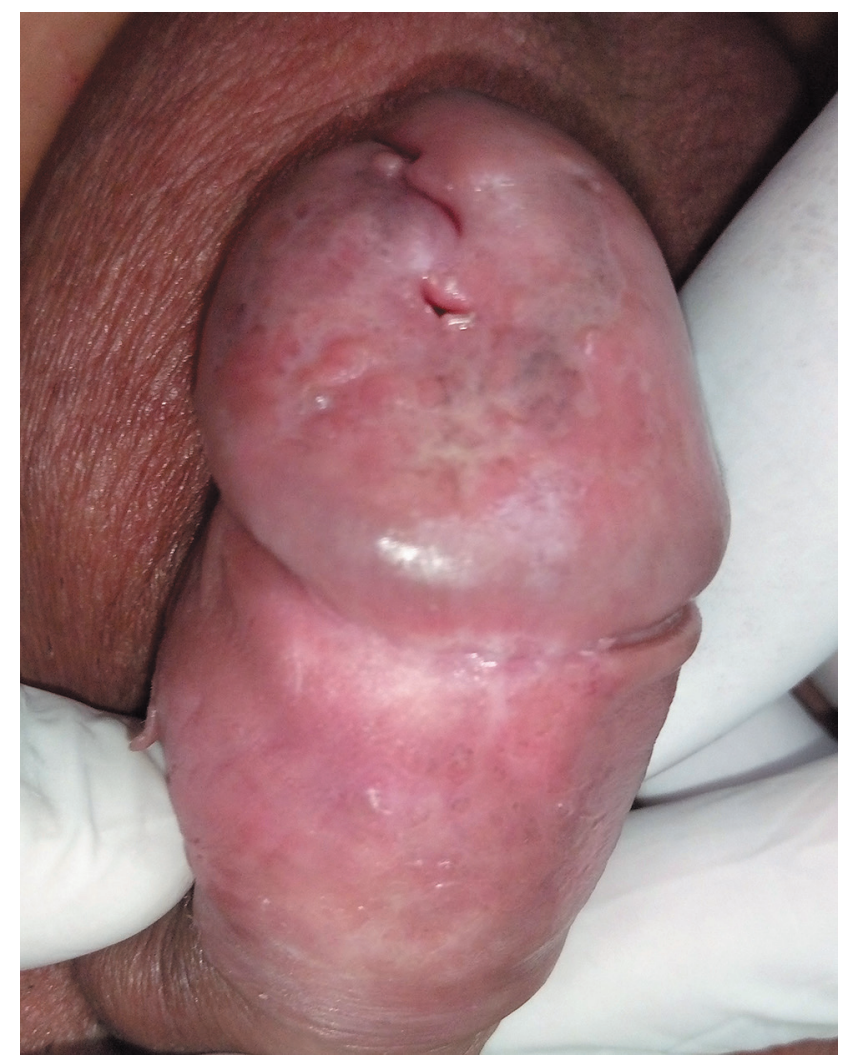

Fig. 3 Healed ulcers with depressed scars after 6 months of antitubercular treatment. and ethambutol for 2 months followed by isoniazid, rifampicin, and ethambutol for 4 months) and complete healing with depressed scars occurred after 4 months of therapy ( - Fig. $\mathbf{3}$ ).

Pulmonary tuberculosis is a common infection in our part of the world; however, tuberculosis of penis is rarely reported. Tuberculids are cutaneous lesions believed to arise from hypersensitivity to Mycobacterium tuberculosis. Tuberculids are characterized by positive tuberculin test, evidence of present or past tuberculosis, absence of M. tuberculosis in the skin lesions, and response to antitubercular treatment. $^{1}$

In our part of the world, prevalence of tuberculosis is still high, and the existence of papulonecrotic tuberculid in penile area is possible. Tuberculosis of glans penis must be excluded if any patient present with unhealthy, nonhealing ulcer in penis.

\section{Conflict of Interest}

None declared.

\section{References}

1 Gangalakshmi C, Sankaramahalingam. Tuberculosis of glans penis- a rare presentation. J Clin Diagn Res 2016;10(12): PD05-PD06 